
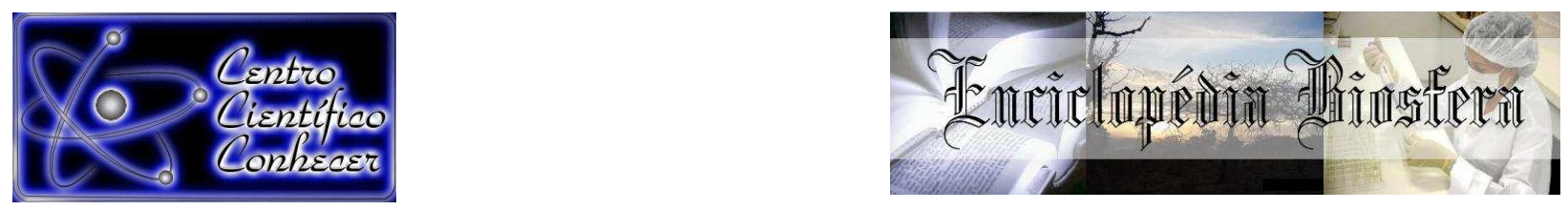

\title{
FONTE ALTERNATIVA DE POTÁSSIO E SEU EFEITO NOS ATRIBUTOS QUÍMICOS DE UM LATOSSOLO VERMELHO DISTRÓFICO CULTIVADO COM CAFEEIRO CATUAÍ
}

\author{
Angélica Araújo Queiroz', Kamila Maria Elias Figueiredo², Renato Barbosa \\ Miranda ${ }^{2}$, Tiago Tadeu Nunes Borges ${ }^{3}$ \\ ${ }^{1}$ Professora doutora do Instituto Federal do Triângulo Mineiro, campus \\ Uberlândia (angelica@iftm.edu.br), Uberlândia-MG,Brasil. \\ ${ }^{2}$ Engenheiro agrônomo graduado pelo Instituto Federal do Triângulo Mineiro, \\ campus Uberlândia. \\ ${ }^{3}$ Engenheiro Agrônomo graduado pela Universidade Federal de Viçosa.
}

Recebido em: 08/04/2017 - Aprovado em: 10/06/2017 - Publicado em: 20/06/2017 DOI: 10.18677/EnciBio_2017A55

\section{RESUMO}

A baixa fertilidade natural dos solos brasileiros e consequentemente a alta demanda por fertilizantes, torna-se um cenário importante na busca por fontes alternativas de nutrientes essenciais para o desenvolvimento das culturas. Assim, este ensaio teve como objetivo avaliar uma fonte alternativa de potássio e seu efeito em atributos químicos de um Latossolo Vermelho distrófico cultivado com cafeeiro, cultivar Catuaí. O experimento foi instalado e conduzido no IFTM, campus Uberlândia, no município de Uberlândia-MG. O delineamento experimental utilizado foi o de blocos ao acaso com cinco tratamentos e quatro repetições, totalizando 20 parcelas. Cada parcela foi constituída por seis plantas. Os tratamentos foram compostos por 5 doses da fonte fonolito $\left(\mathrm{SiO}_{2} 52 \%-\mathrm{K}_{2} \mathrm{O}-8 \%\right)$, sendo estas: $0,100,200,300$ e 400 $\mathrm{kg} / \mathrm{ha}$ correspondentes a 0, 40, 80, 120 e $160 \mathrm{~g} /$ planta aplicadas na projeção da copa do cafeeiro. Foram avaliados o pH em água $(1: 2,5)$ do solo $(0 \mathrm{a} 20 \mathrm{~cm})$ aos 50 e 150 dias após a fertilização e os teores de $\mathrm{P}, \mathrm{Ca}^{2+} ; \mathrm{Mg}^{2+}, \mathrm{K}^{+}$. Após 150 dias da aplicação do Fonolito obteve-se incremento nos valores de $\mathrm{pH}$ e nos teores de fósforo do solo, não sendo observada nenhuma alteração nos teores de potássio do solo, e demais atributos avaliados.

PALAVRAS-CHAVE: Coffea arabica. Fertilizantes. Fontes potássicas.

\section{ALTERNATIVE SOURCE OF POTASSIUM AND THE EFFECT ON CHEMICAL ATTRIBUTES OF A DYSTROPHIC RED LATOSOL CULTIVATED WITH COFFEE}

\begin{abstract}
The low natural fertility of Brazilian soils and consequently the great demand for fertilizers makes it important to search for alternative sources of essential elements for the development of crops. The high demand of potassium in agriculture and the reduced Brazilian mineral reserve, source of this nutrient, makes important the search for alternative sources of potassium. Thus, this research aimed to evaluate an alternative source of potassium and its effect on chemical attributes of a dystrophic Red Latosol cultivated with coffee, cultivar Catuaí. The experiment was installed and conducted at IFTM, campus Uberlândia, in the municipality of Uberlândia-MG. The
\end{abstract}


experimental design was a randomized block design with five treatments and four replications, totaling 20 plots. Each plot was constituted by six plants. The treatments were composed of 5 doses of the fonolite source $\left(\mathrm{SiO}_{2} 52 \%-\mathrm{K}_{2} \mathrm{O}-8 \%\right)$, being: 0 , $100,200,300$ and $400 \mathrm{~kg} \mathrm{ha}^{-1}$ corresponding to $0,40,80,120$ and $160 \mathrm{~g} \mathrm{plant}^{-1}$ applied in the projection of the coffee canopy. The $\mathrm{pH}$ in water $(1: 2.5)$ of the soil $(0$ to $20 \mathrm{~cm}$ ) was evaluated at 50 and 150 days after fertilization and the contents of $\mathrm{P}, \mathrm{Ca}^{2}$ ${ }^{+}$; $\mathrm{Mg}^{2+}, \mathrm{K}^{+}$. After 150 days of application of Fonolito, $\mathrm{pH}$ values and soil phosphorus levels were increased, with no change in soil potassium content, and others attributes assessed.

KEYWORDS: Coffea arabica. Fertilizers. Potassic sources.

\section{INTRODUÇÃO}

A área cultivada no Brasil com a cultura de café (Coffea arabica e Coffea canephora) totaliza 2,23 milhões de hectares. O estado de Minas Gerais é o maior produtor de café do Brasil, tendo uma previsão de produzir na safra de 2017, 26,8 milhões de sacas de café (CONAB, 2017).

O potássio $(\mathrm{K})$ é um nutriente essencial às plantas, no entanto em solos altamente intemperizados, como os solos que ocorrem na maioria do território brasileiro e especialmente no Cerrado brasileiro, este nutriente se encontra abaixo dos teores adequados, por serem facilmente lixiviados (EMBRAPA, 2013). A elevada exigência de potássio demandada pelas culturas se depara com os teores insuficientes ou relativamente baixos deste nutriente nos solos brasileiros, o que tem proporcionado grande aumento no consumo de adubos potássicos para atender a demanda da agricultura brasileira em Ascenção (NACHTIGALL \& RAIJ, 2005).

Segundo CLEMENTE et al. (2013), a demanda de potássio na cultura do café aumenta com a idade da planta, e é intensificada quando a mesma atinge a maturidade. O potássio além de participar de diversas funções fisiológicas no cafeeiro atua na formação dos frutos e grãos de café, agindo na atividade enzimática na síntese e transporte de carboidratos, atribuindo, assim, maior qualidade de bebida (MANCUSO, 2014).

Os principais fertilizantes potássicos utilizados na agricultura são o cloreto de potássio $(\mathrm{KCl})$, o sulfato de potássio $\left(\mathrm{K}_{2} \mathrm{SO}_{4}\right)$, o nitrato de potássio $\left(\mathrm{KNO}_{3}\right)$ e o sulfato de potássio e magnésio $\left(\mathrm{K}_{2} \mathrm{SO}_{4} \cdot \mathrm{MgSO}_{4}\right)$. Dentre essas fontes, $0 \mathrm{KCl}$ representa aproximadamente $95 \%$ de todo o potássio usado na agricultura (GRANJEIRO \& CECÍLIO FILHO, 2006).

A utilização de fontes alternativas de fornecimento de sais de potássio as culturas, e consequente redução na demanda do cloreto de potássio tem evidenciado o uso de rochas com elevados teores deste nutriente em sua constituição. Sendo assim, o desenvolvimento de fertilizantes derivados de rochas brasileiras beneficiaria toda a cadeia produtiva (RIBEIRO et al., 2010), além de ser uma alternativa ao cultivo orgânico.

O fonolito é uma rocha vulcânica encontrada em solos brasileiros. Esta rocha possui em sua composição mineralógica o predomínio de feldspato potássico, feldspato plagioclásios e feldspatóides. A rocha fonolito é empregada principalmente, pelas indústrias cerâmicas como um fundente, devido ao elevado teor de óxidos alcalinos (TEIXEIRA et al., 2015).

O fonolito é apontado como uma fonte alternativa no fornecimento de potássio na agricultura. Este mineral pode apresentar características desejáveis como a elevação do potencial hidrogeniônico do solo, fornecimento de nutrientes além de 
possuir efeito residual prolongado reduzindo assim as perdas de nutrientes por lixiviação (TEIXEIRA et al., 2012).

Visto que a importação de fertilizantes onera os custos da produção agrícola e a busca por alternativas ao cultivo orgânico, torna se importante o estudo da utilização de fontes alternativas com matérias primas, minimizando assim os gastos com importação e ampliando as alternativas para o mercado consumidor.

Assim o objetivo do presente trabalho foi avaliar o efeito do uso do pó de rocha fonolito como fonte alternativa de potássio e seu efeito em atributos químicos de um Latossolo Vermelho distrófico cultivado com cafeeiro cultivar catuaí.

\section{MATERIAL E METODOS}

O trabalho foi conduzido na Fazenda Sobradinho do Instituto Federal do Triângulo Mineiro (IFTM) cujo solo é um Latossolo Vermelho distrofico (LVd) e de textura argilosa, de acordo com a classificação da EMBRAPA (2013).

A fazenda está localizada no município de Uberlândia-MG, com altitude de aproximadamente entre 650 a $660 \mathrm{~m}$. A pluviosidade média da região é de aproximadamente de $1580 \mathrm{~mm}$, temperatura média anual de $22,30{ }^{\circ} \mathrm{C}$ e clima tropical de altitude segundo a classificação de Köpen.

O estudo foi conduzido em área pré-estabelecida de cafezal, variedade Catuaí, com quatro anos de idade, plantado com espaçamento de quatro metros entre linhas e um metro entre plantas. O delineamento experimental foi o de blocos ao acaso com cinco tratamentos e quatro repetições, totalizando 20 parcelas, cada parcela experimental foi constituída por uma linha com seis plantas, sendo adotadas como plantas úteis as quatro centrais, totalizando 120 plantas.

A fonte alternativa de $\mathrm{K}$ estudada foi à rocha fonolito, produto obtido junto à Mineração Curimbaba, localizada na região de Poços de Caldas-MG. A rocha utilizada é formada principalmente por feldspatos alcalinos e feldspatóide, ou seja, microclínio e ortoclásio $\left(\mathrm{KAISi}_{3} \mathrm{O} 8\right)$, sanidina $\left[(\mathrm{Na}, \mathrm{K}) \mathrm{AlSi}_{3} \mathrm{O}_{8}\right)$ e nefelina $\left.(\mathrm{Na}, \mathrm{K}) \mathrm{AlSiO}_{4}\right)$ e apresenta a seguinte composição: $\mathrm{K}_{2} \mathrm{O}$ Total (8-10\%), SiO 2 Total (52-54\%) e pH 9.

Anteriormente a aplicação dos tratamentos foi realizada a amostragem de solo para a caracterização química do solo na profundidade de $0-20 \mathrm{~cm}$, que apresentou o seguinte resultado: $\mathrm{pH}$ em $\mathrm{H}_{2} \mathrm{O}(1: 2,5)=5,7$; $\mathrm{P}$ Mehlich-1 = 31,1 $\mathrm{mg} / \mathrm{dm}^{3} \mathrm{~K}=135 \mathrm{mg} / \mathrm{dm}^{3} \mathrm{Ca}=2,5 \mathrm{Cmolc}_{\mathrm{c}} / \mathrm{dm}^{3} ; \mathrm{Mg}=1,7 \mathrm{Cmol} / \mathrm{dm}^{3}$ de acordo com a metodologia adotada pela EMBRAPA (2011).

Os tratamentos foram compostos pelas diferentes doses da fonte fonolito sendo estas: $0,100,200,300$ e $400 \mathrm{~kg} \mathrm{ha}^{-1}$, correspondentes a, 0, 40, 80, 120 e 160 $\mathrm{g}$ planta $^{-1}$ aplicadas na projeção da copa do cafeeiro, aplicação foi feita manualmente, na projeção da copa do cafeeiro. Foram analisados os teores de $\mathrm{Ca}$, $\mathrm{Mg}, \mathrm{K}$ e $\mathrm{P}$ aos 150 dias e valores de $\mathrm{pH}$ aos 50 e 150 dias, de acordo com a metodologia da EMBRAPA (2011).

Os dados obtidos foram submetidos às análises estatísticas, as médias foram comparadas com o teste de $\mathrm{F}$ e teste de Tukey nos níveis de significância de $5 \%$ de probabilidade e realizada a análise de regressão para as doses estudadas, utilizando o programa estatístico ASSISTAT (SILVA \& AZEVEDO, 2016).

\section{RESULTADOS E DISCUSSÃO}

De acordo com a Tabela 1, pode-se verificar que não houve diferença significativa para os teores de $\mathrm{P}, \mathrm{K}, \mathrm{Ca}, \mathrm{Mg}$ e $\mathrm{pH}$ no solo aos 150 dias após a fertilização. 
TABELA 1- Resumo do quadro de análise de variância, para os teores de $P, K$ e os valores de $\mathrm{pH}$ no solo aos 150 dias após a fertilização.

\begin{tabular}{|c|c|c|c|c|c|c|}
\hline \multirow[b]{2}{*}{ FV } & \multirow[b]{2}{*}{ GL } & \multicolumn{5}{|c|}{ F CALCULADO } \\
\hline & & $\begin{array}{c}\text { Fósforo } \\
(\mathrm{P})\end{array}$ & $\begin{array}{l}\text { Potássio } \\
\text { (K) }\end{array}$ & $\begin{array}{l}\text { Cálcio } \\
\text { (Ca) }\end{array}$ & $\begin{array}{l}\text { Magnésio } \\
(\mathrm{Mg})\end{array}$ & $\mathrm{pH}$ \\
\hline $\begin{array}{l}\text { Doses de } \\
\text { fonolito }\end{array}$ & & $0.7055^{\mathrm{ns}}$ & $0.4176^{\mathrm{ns}}$ & $0.9043^{\mathrm{ns}}$ & $0.4849^{\mathrm{ns}}$ & $0.0196^{\mathrm{ns}}$ \\
\hline Tratamento & 4 & & & & & \\
\hline Resíduo & 15 & & & & & \\
\hline
\end{tabular}

Os resultados do presente trabalho corroboram com os resultados encontrados por QUEIROZ et al. (2016), que estudando esta mesma fonte, encontraram respostas negativas no desenvolvimento de mudas de mogno. Por apresentar baixa solubilidade, a fonte estudada, não disponibiliza o K. TEIXEIRA et al. (2015), estudando o comportamento térmico do fonolito, verificaram que a disponibilidade de potássio em amostras de fonolito calcinadas a 600 e $1200^{\circ} \mathrm{C}$ foi de aproximadamente $2 \%$, ou seja, muito abaixo da disponibilidade que as fontes convencionais apresentam.

$\mathrm{O} \mathrm{pH}$ do solo é utilizado como indicativo das condições gerais da fertilidade, pois reflete indiretamente 0 teor de bases existentes no solo, e de acordo com a tabela 2, pode-se observar que não houve diferença para os valores de $\mathrm{pH}$ nas duas épocas de amostragem, e consequentemente não houve aumento nos teores de $\mathrm{Ca}$ e Mg no solo.

Estudos que buscam uma alternativa ao calcário, objetivando corrigir a acidez do solo e reduzir possíveis impactos ao ambiente através do reaproveitamento de resíduos industriais tem sido cada vez mais pesquisado, NOGUEIRA (2012) estudando diferentes doses de até $125 \%$ da necessidade de calagem, de escória de siderurgia, óxido de magnésio e calcário em dois solos sob o cultivo de café arábica, verificou que no Latossolo Vermelho Amarelo, com textura argilosa, os valores de $\mathrm{pH}$ do solo foram maiores quando utilizado o óxido de magnésio, que diferiu dos demais corretivos estudados. Mas não ocorreu diferença estatística entre os corretivos quando aplicado em um Latossolo Vermelho Amarelo de textura media., mostrando assim, a influência da capacidade tampão de um solo sobre o desempenho dos corretivos.

Comportamento semelhante com o uso de fontes alternativas na elevação do pH foi constatado também por MELO et al., (2012), que estudando diferentes doses de basalto moído em um Latossolo Amarelo distrófico, obtiveram valores de $\mathrm{pH}$ do solo elevados com o acréscimo das doses.

O desempenho agronômico destas rochas pode variar também de acordo com o material de origem. SILVA (2012), observando o comportamento de diferentes dosagens de rochas silicaticas moídas sob os atributos químicos do solo nas dosagens de 200, 400 e $600 \mathrm{~kg}_{\text {de }} \mathrm{K}_{2} \mathrm{O} \mathrm{ha}^{-1}$ incubadas por 100 dias, constatou que a rocha ultramáfica e o subproduto de chapada foi as rochas que mais influenciou a elevação do $\mathrm{pH}$ do solo quando comparadas as rochas flogopitito, brecha e biotita xisto, enquanto o subproduto de mineração foi a rocha que mais liberou potássio. 
TABELA 2- Medias do pH aos 50 e aos 150 dias em função das doses de fonolito, aplicadas no solo.

\begin{tabular}{ccc}
\hline Doses $\mathbf{~ g ~ h ~}$ $^{-1}$ & pH aos $\mathbf{5 0}$ dias & pH aos150 dias \\
\hline 400 & 5,3 & 5,8 \\
300 & 5,3 & 6,1 \\
200 & 5,2 & 5,8 \\
100 & 5,5 & 5,7 \\
0 & 5,0 & 5,7 \\
\hline
\end{tabular}

Pode-se verificar, pela Tabela 3, que os teores de $\mathrm{Ca}$ e $\mathrm{Mg}$ estão dentro da faixa de disponibilidade considerada média e os teores de $\mathrm{K}$ e $\mathrm{P}$ considerado muito bom, de acordo com RIBEIRO et al. (1999). Mesmo não apresentando diferença significativa para os atributos químicos analisados, pode-se verificar que houve um aumento nos teores de P e K no solo, após a aplicação do fonolito. Inicialmente, os teores de $P$ que era de $31,1 \mathrm{mg} / \mathrm{dm}^{3}$ aumentaram para valores de até $66,7 \mathrm{mg} / \mathrm{dm}^{3}$ e o K que passou de $135 \mathrm{mg} / \mathrm{dm}^{3}$ para até $268 \mathrm{mg} / \mathrm{dm}^{3}$ na dose de $100 \mathrm{~kg} \mathrm{ha}^{-1}$ de fonolito.

TABELA 3- Medias dos teores de $\mathrm{Ca}, \mathrm{Mg}, \mathrm{P}$ e $\mathrm{K}$ aos 150 dias em função das doses de fonolito, aplicadas no solo.

\begin{tabular}{cccccc}
\hline Doses kg ha & -1 & Ca & Mg & P & K \\
\hline \multicolumn{2}{c}{${\text { Cmolc } / \mathrm{dm}^{3}}^{2}$} & & $\mathrm{mg}^{3} \mathrm{dm}^{3}$ \\
\hline 400 & 2,5 & 1,1 & 65,7 & 218 \\
300 & 2,9 & 1,2 & 66,7 & 268 \\
200 & 2,0 & 1,1 & 37,6 & 223 \\
100 & 2,5 & 0,9 & 45,1 & 213 \\
0 & 2,4 & 1,0 & 47,9 & 186 \\
\hline
\end{tabular}

$\mathrm{O}$ não incremento nos teores de $\mathrm{P}$, pode estar relacionado com os valores encontrados de pH no solo. De acordo com PREZOTTI \& MARTINS (2012), o silicato tem papel importante na diminuição da adsorção de $P$ em solos tropicais, visto que o íon silicato compete com o íon fosfato pelos mesmos sítios de adsorção na superfície dos minerais, tornando este último mais disponível às plantas.

Estudos avaliando a aplicação de fonolito na cultura do café (MANCUSO, 2014) constataram teores de Si presente nas folhas, demonstrando que a fonte é eficiente na liberação de Si na solução do solo, ficando assim disponível para as plantas. Estudando também fontes alternativas de fertilizante PRADO \&FERNANDES (2001), constataram que o uso de escória de siderurgia incrementou os níveis de $\mathrm{P}$ disponível do solo, enquanto que a aplicação de calcário não apresentou uma relação significativa.

De acordo com TEIXEIRA et al. (2015) a rocha fonolito calcinada, pode ser uma fonte alternativa de $\mathrm{K}$, porém como liberação lenta deste nutriente, que conforme o tratamento térmico dado à rocha, esta pode chegar a ter $2 \%$ de $\mathrm{K}$ liberado no solo, o que corrobora com os resultados encontrados no presente trabalho, uma vez que os teores de $\mathrm{K}$ no solo não diferenciaram estatisticamente em função das doses de fonolito testadas (Tabela 1).

Quanto aos teores de $\mathrm{Ca}$ e Mg, TEIXEIRA et al. (2012) citam que a fonte fonolito tem em a composição silício, cálcio, ferro e manganês, que são requerido pelas plantas, porém mais estudos são necessários para se conhecer a 
disponibilidade destes nutrientes pela fonte, este fato pode explicar a ausência de influência das doses crescentes testadas nos teores de $\mathrm{Ca}$ e $\mathrm{Mg}$ encontrados.

Segundo CARVALHO (2013) os pós de rocha silicatadas são, essencialmente, fontes multinutrientes de liberação lenta. Dessa forma, a tendência geral é que estas fontes sejam menos responsivas, em curto prazo, que os fertilizantes de alta solubilidade ou que os termofertilizantes em condições de solos altamente deficientes em nutrientes, fato este que pode explicar a não resposta nos atributos químicos avaliados pelo presente trabalho.

Outro fator que pode ter contribuído para os teores de $\mathrm{K}$ encontrados não ter sofrido alterações, pode ser devido à forma de obtenção da fonte fonolito, que é obtida através da moagem da rocha in natura, sendo que resultados positivos com o uso de pó de rochas foram detectados após processos químicos ou tratamentos térmicos, com o intuito de aumentar a solubilização dos nutrientes, quando a rocha é aplicada ao solo.

MANCUSO (2014) constatou resultados positivos na utilização da rocha fonolito in natura na cultura do café, onde obteve incrementos semelhantes aos obtidos pelo $\mathrm{KCl}$ quando adotando uma taxa de $250 \mathrm{~kg} \mathrm{~K}_{2} \mathrm{O} \mathrm{ha}{ }^{-1}$, representando uma aplicação de $1560 \mathrm{~kg} \mathrm{ha}^{-1}$ da fonte fonolito. Dosagem esta superior à estudada no presente trabalho, evidenciando assim uma limitação quanto à logística, já que a quantidade necessária para efeitos satisfatórios iria elevar os custos com 0 transporte, sendo mais viável o seu emprego em áreas próximas as usinas de produção.

\section{CONCLUSÕES}

A fonte estudada, fonolito, não promoveu modificações nos atributos químicos do solo avaliados, $\mathrm{K}, \mathrm{Ca}, \mathrm{Mg}, \mathrm{P}$ e $\mathrm{pH}$.

\section{AGRADECIMENTOS}

Ao IFTM, campus Uberlândia pelo apoio à realização do trabalho e ao conselho nacional de pesquisa CNPQ pela concessão da bolsa.

\section{REFERÊNCIAS}

CARVALHO, A. M. X. ROCHAGEM: UM NOVO DESAFIO PARA O MANEJO SUSTENTÁVEL DA FERTILIDADE DO SOLO. In: SILVA, J. C. da; SILVA, A. A. S.; ASSIS, R. T. de (Org.). Sustentabilidade produtiva e inovação no campo. Uberlândia: Composer, 117-132p. 2013.

CONAB (COMPANIA NACIONAL DE ABASTECIMENTO). Acompanhamento safra brasileira de café, v. 4 - Safra 2017, n.1 - Primeiro Levantamento, p. 1-98, 2017, Disponível em: http://www.conab.gov.br/OlalaCMS/uploads/arquivos/17_01_17_14_51_54_boletim_ cafe_-_janeiro_de_2017.pdf >. Acesso em: 03 de maio de 2017.

CLEMENTE, J. M.; MARTINEZ, H. E. P; ALVES, L. C.; LARA, M. C. R. Effect of N and $\mathrm{K}$ doses in nutritive solution on growth, production and coffee bean size. Revista Ceres, v.60, n.2, pp.279-285. 2013. Disponível em: <http://dx.doi.org/10.1590/S0034-737X2013000200018>. doi: 10.1590/S0034737X2013000200018. 
EMPRESA BRASILEIRA DE PESQUISA AGROPECUÁRIA. Centro Nacional de Pesquisa de Solo. Manual de métodos de analise de solo $2^{\mathrm{a} e d i c ̧ a ̃ o . ~ B r a s i ́ l i a . ~} 225 \mathrm{p}$. 2011.

EMPRESA BRASILEIRA DE PESQUISA AGROPECUÁRIA. Centro Nacional de Pesquisa de Solo. Sistema Brasileiro de Classificação de Solos $3^{a}$ edição. Brasília: EMBRAPA, SIBCS. 353p. 2013.

GRANGEIRO, L. C.; CECÍLIO FILHO, A. B. Características de produção de frutos de melancia sem sementes em função de fontes e doses de potássio. Horticultura Brasileira, v. 24, n. 4, p. 451- 454, 2006. Disponível em: <http://dx.doi.org/10.1590/S0102-05362006000400011>.doi: 05362006000400011.

MANCUSO, M. A. C.; SORATTO, R.P.; CRUSCIOL, C.A.C.; CASTRO, G.S.A. Effect of potassium sources and rates on Arabica coffee yield, nutrition, and macronutrient export. Revista Brasileira de Ciência do Solo, v. 38, n. 5, p. 1448-1456, 2014. Disponível em: <http://dx.doi.org/10.1590/S0100-06832014000500010>. doi: 10.1590/S0100-06832014000500010.

MELO, V.F.; UCHOA, S. C. P.; DIAS, F.O.; BARBOSA, G. F. Doses de basalto moído nas propriedades químicas de um Latossolo Amarelo distrófico da savana de Roraima. Acta Amazonica. 2012, v.42, n.4, p.471-476. ISSN 0044-5967. Disponível em: http://dx.doi.org/10.1590/S0044-59672012000400004. doi: 10.1590/S004459672012000400004.

NACHTIGALL, G.R.; RAIJ, B.van. Análise e interpretação de potássio no solo. In: YAMADA, T.; ROBERTS, T.L., eds. Potássio na agricultura brasileira. Piracicaba, Associação Brasileira para a Pesquisa da Potassa e do Fosfato, p.93-118. 2005.

NOGUEIRA, N. O. TOMAZ, M. A ; ANDRADE, F. V.; REIS, E. F. DOS; BRINATE, S. $V$. B. Influência da aplicação de dois resíduos industriais nas propriedades químicas de dois solos cultivados com café arábica. Revista Ciência Agronômica. v.43, n.1, p.11-21. 2012. Disponível em: <http://dx.doi.org/10.1590/S180666902012000100002> . doi: 10.1590/S1806-66902012000100002.

PRADO, R. M.; FERNANDES, F. M. Efeito da escória de siderurgia e calcário na disponibilidade de fósforo de um Latossolo Vermelho-Amarelo cultivado com canade-açúcar. Pesquisa Agropecuária Brasileira. v.36, n.9, p.1199-1204. 2001. Disponível em: < http://dx.doi.org/10.1590/S0100-204X2001000900014>. doi:10.1590/S0100-204X2001000900014.

PREZOTTI, L. C.; MARTINS, A.G. Efeito da escória de siderurgia na química do solo e na absorção de nutrientes e metais pesados pela cana-de-açúcar. Revista Ceres. v.59, n.4, p.530-536. 2012. Disponível em: <http://www.scielo.br/scielo.php?script=sci_arttext\&pid=S0034-

737X2012000400014\&lng=pt\&nrm=iso>. - doi: http://dx.doi.org/10.1590/S0034737X2012000400014. 
QUEIROZ, A. A. ;ANGELINI, M. R.; RODRIGUES, H.F. FONOLITO NA PRODUÇÃO DE MUDAS DE MOGNO. Enciclopédia Biosfera, v.13 n.23; 637 -645 p. 2016 Disponível em: <http://www.conhecer.org.br/enciclop/2016a/agrarias/fonolito.pdf> Acesso em: 02 maio de 2017. doi: 10.18677/Enciclopedia_Biosfera_2016_056.

RIBEIRO, A. C.; GUIMARÃEZ, P. T.; ALVAREZ, V. H. Recomendação para o uso de corretivos e fertilizantes para o Estado de Minas Gerais. 5a aproximação. Viçosa, MG, CFSEMG, 1999.

RIBEIRO, L. da S.; SANTOS, A. R. dos; SOUZA, L. F. da S.; SOUZA, J. S.. Rochas silicáticas portadoras de potássio como fontes do nutriente para as plantas solo. Revista Brasileira de Ciência do Solo. v. 34, n.3, p.891-897. 2010. Disponível em: <http://dx.doi.org/10.1590/S0100-06832010000300030>. doi:10.1590/S010006832010000300030.

SILVA, D. R. G.; MARCHI, G.; SPEHAR, C. R.; GUILHERME, L.R.G.; REIN, T. A.; SOARES, D.A.; ÁVILA, F. W. Characterization and nutrient release from silicate rocks and influence on chemical changes in soil. Revista Brasileira de Ciência Solo. v.36, n.3, p.951-962. 2012. Disponível em: <http://dx.doi.org/10.1590/S010006832012000300025>. doi:10.1590/S0100-06832012000300025.

SILVA, F. de A. S.; AZEVEDO, C. A. V. de. The Assistat Software Version 7.7 and its use in the analysis of experimental data. African Journal of Agricultural Research, v. $11, \quad$ n. $39, \quad$ p. $3733-3740,2016$. Disponível em: <http://www.assistat.com/indexp.html> doi: http://dx.doi.org/10.5897/AJAR2016.111522.

TEIXEIRA, A. M. S.; SAMPAIO, J. A.; GARRIDO, F.M.S; MEDEIROS, M.E. Avaliação da rocha fonolito como fertilizante alternativo de potássio. Holos, v.5, p.21-23, 2012. Disponível em:

<http://www2.ifrn.edu.br/ojs/index.php/HOLOS/article/view/1102/593>. Acesso em:16 fev. 2016. doi: 10.15628/holos.2012.1102.

TEIXEIRA, A. M. S.; GARRIDOA, F. M. S.; MEDEIROS, M. E.; SAMPAIO, J. A. Effect of thermal treatments on the potassium and sodium availability in phonolite rock powder. International Journal of Mineral Processing, v. 145, p. 57-65, 2015. Disponível em: <http://www.sciencedirect.com/science/article/pii/S030175161530003X >. Acesso em: 30 de mar. 2017. doi: http://dx.doi. org/10.1016/j.minpro.2015.07.002. 